\title{
Finishing the finished human chromosome 22 sequence
} Charlotte G Cole ${ }^{\mathfrak{x}^{*}}$, Owen T McCann ${ }^{\alpha^{*}}$, John E Collins ${ }^{*}$, Karen Oliver ${ }^{*}$, David Willey*, Susan M Gribble*, Fengtang Yang*, Karen McLaren*, Jane Rogers*, Zemin Ning*, David M Beare* and Ian Dunham* ${ }^{*}$

\begin{abstract}
Addresses: *The Wellcome Trust Sanger Institute, Wellcome Trust Genome Campus, Hinxton, Cambridge, CB10 1SA, UK. "EMBL-European Bioinformatics Institute, Wellcome Trust Genome Campus, Hinxton, Cambridge, CB10 1SD, UK.
\end{abstract}

These authors contributed equally to this work.

Correspondence: Ian Dunham. Email: dunham@ebi.ac.uk

Published: I 3 May 2008

Genome Biology 2008, 9:R78 (doi:10.1 186/gb-2008-9-5-r78)

The electronic version of this article is the complete one and can be found online at http://genomebiology.com/2008/9/5/R78
Received: 19 February 2008

Revised: 10 April 2008

Accepted: 13 May 2008

(C) 2008 Cole et al.; licensee BioMed Central Ltd.

This is an open access article distributed under the terms of the Creative Commons Attribution License (http://creativecommons.org/licenses/by/2.0), which permits unrestricted use, distribution, and reproduction in any medium, provided the original work is properly cited.

\begin{abstract}
Background: Although the human genome sequence was declared complete in 2004, the sequence was interrupted by $34 \mathrm{I}$ gaps of which 308 lay in an estimated approximately $28 \mathrm{Mb}$ of euchromatin. While these gaps constitute only approximately $1 \%$ of the sequence, knowledge of the full complement of human genes and regulatory elements is incomplete without their sequences.
\end{abstract}

Results: We have used a combination of conventional chromosome walking (aided by the availability of end sequences) in fosmid and bacterial artificial chromosome (BAC) libraries, whole chromosome shotgun sequencing, comparative genome analysis and long PCR to finish 8 of the II gaps in the initial chromosome 22 sequence. In addition, we have patched four regions of the initial sequence where the original clones were found to be deleted, or contained a deletion allele of a known gene, with a further $126 \mathrm{~kb}$ of new sequence. Over $1.018 \mathrm{Mb}$ of new sequence has been generated to extend into and close the gaps, and we have annotated 16 new or extended gene structures and one pseudogene.

Conclusion: Thus, we have made significant progress to completing the sequence of the euchromatic regions of human chromosome 22 using a combination of detailed approaches. Our experience suggests that substantial work remains to close the outstanding gaps in the human genome sequence.

\section{Background}

The completion of the human genome sequence was the culmination of the 15 year Human Genome Project. The finished sequence contained $2.85 \mathrm{~Gb}$ and was estimated to cover $99 \%$ of the euchromatin [1]. Thus far the human genome is the only gigabase scale sequence to obtain the necessary high accuracy and near completeness to be published as a 'finished' standard, although the mouse genome is expected to join it soon. However, although significant efforts were made to obtain maximum continuity, the sequence was interrupted 
by 341 gaps. Of these, 308 gaps covered approximately $28 \mathrm{Mb}$ of euchromatin while the remainder represented the heterochromatin, chiefly centromeres and telomeres. While finishing of the sequence was a major milestone, for completists there remain the nagging questions of whether it is possible to close the gaps, and what lies in those missing sequences.

The process of sequencing the human genome was undertaken using the two approaches of whole genome shotgun [2] and map based clone sequencing [3]. However, only the clone-based strategy, which utilized genome maps and large insert clones, allowed ready application of directed strategies for completion of the sequence [1]. The clone-based strategy involved building contiguous maps of the human chromosomes in large-insert cloning vectors such as bacterial artificial chromosomes (BACs), resolved at a local level by restriction enzyme fingerprinting and ordered and orientated with respect to longer range maps of the genome [4,5]. Individual BACs were then selected from the maps to create a set of clones that minimally covered the genome for sequencing. In the first instance the tilepath BACs were subjected to shotgun sequencing and assembled to produce the draft quality genome sequence. Progressing from this point to a complete sequence by the process of finishing required two major components: first, the maps of clones required completing so that substrates were available for sequencing; and second, the sequence within each clone required refining to the highest level of accuracy with no gaps. Thus, gaps in the genome sequence could be of three kinds. There could be gaps within individual clone sequences where either sequence could not be determined, or there was ambiguity or error in the base call (sequence gaps/errors) [6]. There could be gaps where no clone was available from the map for sequencing, including, but not restricted to, heterochromatic and segmental duplicated regions (map gaps) [7]. The third type of gap would result from a problem with the shotgun assembly or with the underlying BAC, such as a deletion resulting in a false join within the sequence (assembly or insertion/deletion errors) $[6,8]$. Quality assessments of the finished human genome sequence suggested that sequence gaps/errors were likely to occur at a rate an order of magnitude lower than the rate of human polymorphism $(<1 / 10 \mathrm{~kb})$, while mis-assembly or insertion/deletion errors were likely to be relatively few $[1,6,9]$, although the precise number remained to be established at all resolutions. In addition, because of the local nature of the sequence assembly for each clone in the clonebased sequence strategy, sequence or mis-assembly gaps were unlikely to affect substantial regions. On the other hand, the number of map gaps was well established and the missing sequence at each gap was known to be on the order of $90 \mathrm{~kb}$ on average.

Therefore, to obtain a complete reference human genome sequence requires identification and sequencing of new clones for map gaps and finding and addressing each base ambiguity or error. This would entail a substantial genome curation activity designed to improve the coverage and accuracy of the sequence. In addition, the current reference sequence is a mosaic of clone sequences derived from more than eight individuals. For genes it would be desirable that the allele in the reference sequence is as far as possible representative of the functional form. For instance, the initial chromosome 22 sequence contained a deletion allele of the CYP2D6 gene [10]. Although this form is reasonably common in European populations, it would be preferable to have a complete version as the reference. Furthermore, in certain regions where there is extensive polymorphism, such as the human leukocyte antigen (HLA) locus, there are arguments for maintaining alternative versions of the common haplotype sequences [11].

Chromosome 22 was the first human chromosome to reach finished sequence standard [8]. On initial publication the sequence comprised 12 contiguous segments spanning 33.4 $\mathrm{Mb}$ of 22q (Figure 1) and included known centromeric and subtelomeric heterochromatin repeats at either end. Four of the map gaps were located in 22q11 in regions associated with the segmental duplications involved in low copy repeats (LCRs) on chromosome 22 (LCR22; here referred to as gaps A-D; Figure 1) [8,12-14]. The remaining seven gaps were located in the $\mathrm{G}+\mathrm{C}$ rich region of 22q13.3 (gaps 1-7; Figure 1) and are not obviously associated with copy number variations (CNVs) in the latest CNV data [15], although CNVs occurring in the gaps would not have been detected.

Since the initial publication we have been working towards closing these gaps, particularly in the 22q13.3 region that was the responsibility of our group in the original chromosome 22 sequencing consortium. Here we report our approaches and progress towards completion of the human chromosome 22 sequence. Our experiences may be pertinent to future efforts to curate the human genome reference sequence.

\section{Results and discussion}

In the following sections we describe our approaches and results towards correction of deletions and closing map gaps on human chromosome 22. The clone library resources used and the information required to decode clone prefixes are provided in Additional data file 1. For reference we have detailed the positions of the gaps and deletions to which we refer on selected genome builds in Additional data files 2 and 3 .

\section{Updating the chromosome 22 sequence to correct deletion alleles and deleted BAC clones}

The initial chromosome 22 sequence included a P1 artificial chromosome (PAC) (RP1-257I20, AL021878) containing a common deletion allele of the CYP2D6 gene [10]. In order to represent this gene in a functional form in the reference sequence, we identified from the clone map a RPCI-4 PAC containing a full copy of CYP2D6 (RP4-669P10, BX247885; 


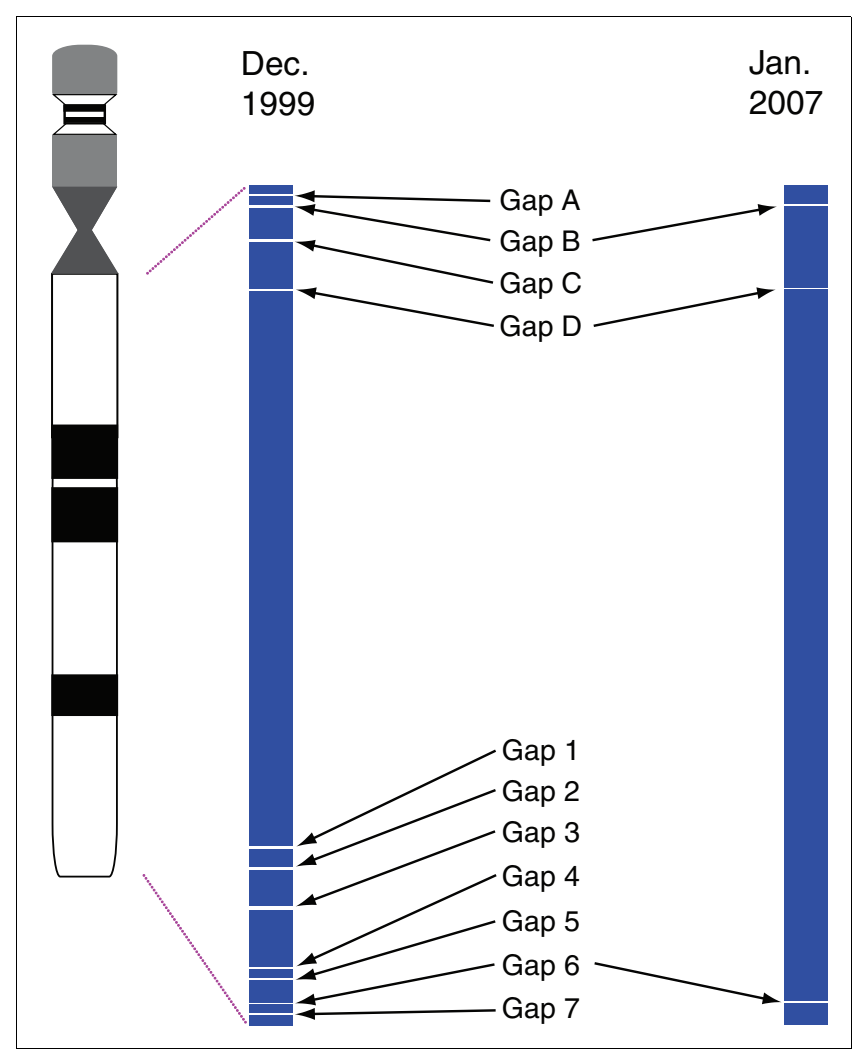

Figure I

Schematic view of sequence contigs (blue boxes) covering chromosome $22 q$ in [8] (1999, left) and in this report (2008, right). Contigs are drawn approximately to scale and shown in relation to a simple representation of the chromosome 22 ideogram. Gaps are indicated by the arrows, and are labeled according to the terminology used in this report.

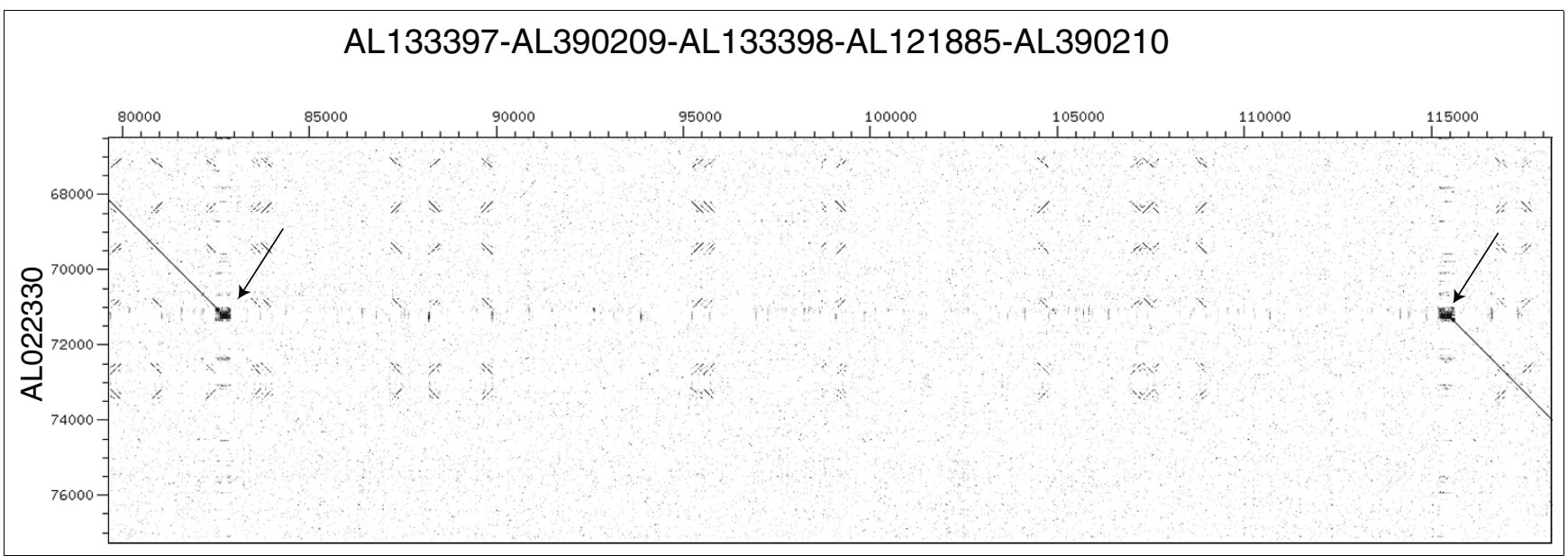

Figure 2

Sequence analysis of deleted BAC CTA-437GI0 (AL022330) as originally finished in [8]. A sequence identity dot plot analysis of the revised sequence of this region ( $x$-axis) against the originally submitted sequence ( $y$-axis) generated using dotter [48]. The revised sequence ( $x$-axis) encompasses $A L I 33397 . I$, AL390209.I, ALI33398.2, ALI21885.22, AL390210.1 assembled as detailed at [49], and numbered from the start of ALI33397.I. Numbering is in basepairs. Arrows indicate the positions of tandem repeated sequences at the boundary of the deletion in this BAC, with a core region of (AAAG) ${ }_{46}$ at the centromeric (left) side and a core of (AAAG) ${ }_{68}$ at the telomeric (right) side. 
be expected for this cloning system, or where one of the paired end sequences was missing, indicating the likely presence of a deletion in the reference sequence relative to the haplotypes present in the WIBR-2 fosmid library $[1,16]$. The presence of deletions in the two RPCI PAC clones contributing to the reference sequence at those points (RP4-633O19, AL022302; RP5-824I19, AL009049) was confirmed by DNA fiber FISH (Figures S2 and S3, respectively, in Additional data file 7). Again, comparison with the known CNV regions did not identify either of these deletions as commonly polymorphic and we chose to identify clones representing the non-deleted forms in both cases for sequencing and inclusion in the reference sequence (G248P-80423F1, BX470187; CITF22-91B8, BX936361 and CITF22-37A6, BX927085; Figures S11 and S12 in Additional data file 7). This sequencing generated an additional approximately $34 \mathrm{~kb}$ and approximately $49 \mathrm{~kb}$ in the reference sequence covering the two deleted regions, respectively. Comparison of the revised sequences with the original deleted clones showed that the deletions in these cases occurred within repetitive sequences (between two copies of MLT1L repeats for RP4-633O19 (AL022302) and between an AluYa5 and a MLT2C1 repeat in RP5-824I19 (ALoo9049)).

Analysis of the $126 \mathrm{~kb}$ of additional sequence generated to cover the four deletions identified that, in addition to CYP2D6, only a single short predicted ribosomal protein gene (G248P-80423F1.C22.1 in BX470187) had previously been missed due to deletion within the bacterial clones used for the initial reference sequence. However, since there is no mRNA or expressed sequence tag mapping with high sequence identity to this gene, it is likely to represent a pseudogene (definitions as in [18]).

\section{Closing map gaps in the chromosome 22 sequence}

During the initial sequencing of chromosome 22q, the construction of physical maps was divided by region between the different members of the consortium [8]. Our initial map gap closure efforts focused on the seven gaps in 22q13, which are numbered gaps 1-7 from centromere to telomere (Figure 1; Figures S4-S10 in Additional data file 7; see Additional data files 2 and 3 for positions of gaps on selected genome builds). In two cases initial mapping had identified clones that, while containing sequences on either side of the gaps, were deleted when analyzed by DNA fiber FISH analysis (data not shown).

The initial mapping process had involved screening of at least 20 genome equivalents of large insert clone libraries (BAC or PAC) and cosmids or fosmids generated from flow sorted chromosomes (see Additional data file 1 for clone libraries used). As a first step to closing the gaps, we identified sequences at the edges of each gap to develop into sequence tagged sites (STSs) and re-screened these libraries. In the case of the flow sorted chromosome 22 fosmid library (CITF22) $[19,20]$ it was clear that additional clones that extended into the gaps had been missed due to false negatives in initial screens in some cases. These clones were incorporated into the maps by restriction enzyme fingerprinting, extension confirmed by DNA fiber FISH and then appropriate clones were chosen to generate sequence extending into the gaps. There subsequently followed further rounds of chromosome walking by developing STSs from the ends of each fosmid and re-screening the CITF22 fosmid library (Figures S4S13 in Additional data file 7; Additional data file 4). With this approach we were able to add additional sequence to extend each gap, ultimately contributing approximately $307 \mathrm{~kb}$ of the total additional sequence generated for gaps 1-7 in 22q13 (42.4\%; Table 1). In addition, we identified two chromosome 22 specific STSs (AFMbo40xdl and WNT7B) for which we had previously been able to identify BAC clones, but had been unable to incorporate these clones into the physical maps at the required stringency of overlap. Re-examination of these data followed by sequencing of two BACs (RP11-435J19, BX511035; RP11-398F12, AL929387), together with an additional PAC (RP6-109B7, AL121672) that had not been finished previously, placed them as extensions into gap 3, adding an additional $137 \mathrm{~kb}$ to the total sequence (18.9\%). As the identification of novel clones from these resources became exhausted, an additional source of fosmid clones became available in the form of the WIBR-2 whole genome library with systematic determination of paired end sequences (Whitehead Institute random genomic fosmid library WIBR-2; Additional data file 1) [1]. The availability of end sequences from this library on the NCBI Trace Archive [21] allowed rapid sequence similarity searching to identify fosmids that extended into the unsequenced gaps. The WIBR2 fosmids contributed an additional approximately $151 \mathrm{~kb}$ (20.9\%) of new sequence. In certain cases we were able to alternate chromosome walking between the CITF22 and WIBR-2 fosmid libraries, for instance while closing gap 5 (Figure S8 in Additional data file 7). Although these walking approaches generated additional chromosome 22 sequence, they were able to close only one of the seven gaps.

In order to make further progress towards completion, we adopted an alternative approach of utilizing long PCR between known sequences at the edges of the gaps, or internal to the gaps. Internal sequence was identified from four sources. First, we searched the chimpanzee genome sequence assembly [22] for orthologous sequences matching the human gap boundaries, and identified chimpanzee contigs extending into the gaps. Second, as part of the process of generating sequences for identification of single nucleotide polymorphisms during the HapMap project, six chromosome equivalents of shotgun sequence reads had been generated from plasmid libraries of flow sorted chromosome 22 from three individuals (whole chromosome shotgun sequence (WCS)) [23]. Nearly 890,000 paired end shotgun sequence reads produced from these plasmids and the CITF22 flow sorted chromosome fosmid library were used to produce a scaffold assembly (see Materials and methods). These shotgun sequence reads were assembled (see Materials and meth- 
Table I

Sources of additional sequence for gaps I-7 in human chromosome 22q I3

\begin{tabular}{lcc}
\hline Source of sequence & Number & Sequence contributed (kb) \\
\hline Flow sorted chromosome 22 fosmids & 14 & 307 \\
Whole genome fosmids & 6 & 151 \\
BAC/PAC & 3 & 137 \\
PCR products & 28 & 129 \\
\hline
\end{tabular}

Note that due to the arbitrary nature of the positions of clipping of submitted sequences, and their contribution to the final golden path, the new sequence contribution numbers are, of necessity, approximated.

ods for details; assembly available at [24]) and the assembly compared to the existing finished sequence. Contigs and scaffolds that extended into the gaps were identified. Third, in one case prior gene annotation [18] indicated the presence of a gene that was only partially contained within existing sequence (gap 1, dJ345P10.c22.4.mRNA). Examination of the cDNA (AKo23424) and expressed sequence tag sequences was used to identify putative exons internal to the gap. Finally, Celera shotgun sequence $[2,25]$ was identified that overlapped with chimp scaffold sequence and extended further into the gap. Sequence from each of these sources predicted to lie internal to the gaps was used together with the known sequence at the edges of gaps to design long PCR oligonucleotide primer pairs that might amplify across the missing sequence (Additional data file 5). Wherever possible, long PCR primers were designed so that the PCR product would encompass the shortest possible span of missing DNA. In addition, where a contig from chimpanzee or flow sorted shotgun sequence extended within a gap, we also aimed to amplify this sequence by long PCR from human DNA in order to cover gaps within the contigs/scaffolds to obtain human sequence in the case of chimp and to obtain independent verification of the unfinished WCS sequence. In one case additional long PCR primers were generated from newly sequenced long PCR products. Long PCR products from a pool of human DNAs (Roche high molecular weight human DNA; see Materials and methods) were obtained in the size range 1.3-20 kb (Figures $\mathrm{S}_{4}-\mathrm{S} 7, \mathrm{~S} 9$, and $\mathrm{S} 10$ in Additional data file 7; Additional data file 5). These products were subcloned into plasmid vectors and sequenced (see Materials and methods). One gap, gap 2, was difficult to amplify across in the final stages of the PCR gap closure, despite expectations that it was $<5 \mathrm{~kb}$. To allow precise refinement of long-PCR conditions based on an accurate estimate of gap size, we performed two-color DNA fiber FISH by labeling long PCR products on either side of the gap, totaling approximately $20 \mathrm{~kb}$ and approximately $6.2 \mathrm{~kb}$, respectively (Figure 3). The remaining gap was thus estimated at $2-3 \mathrm{~kb}$ and was then successfully closed using modified PCR conditions.

At the end of several iterations of these strategies we succeeded in closing six of the seven gaps in 22q13 (Figure 1; Figures $\mathrm{S} 4$-S10 in Additional data file 7). The one remaining gap, gap 6, was found to be bordered by repetitive sequences suf- ficient to prevent further long PCR and exact size determination by fiber FISH. However, a fiber FISH estimate from the flanking clones, together with the addition of $10.5 \mathrm{~kb}$ of sequence by long PCR, suggests that the sequence remaining to be determined is less than $15 \mathrm{~kb}$. In total, we generated 724 $\mathrm{kb}$ of new sequence to close and extend into these gaps, and the final sequences were incorporated into the latest version of the human chromosome 22 reference sequence (Chr_22 release 4 [26], to be incorporated into the next release of the human genome reference sequence: HGRC 37).

During the period of this work, one further gap in 22q11 was closed by chromosome walking in BACs (Figure 1; gap C, AC016026 and AC016027; BA Roe, personal communication). Additional sequence was also produced by the Roe group to shorten the gap between original tile path clones ACo07663 and ACo07731 (gap D, ACo58790, AC024070 and ACo11718). From analysis of end sequences from the WIBR-2 whole genome fosmid library, we have identified a single fosmid clone to close the most centromeric gap in 22q11 (gap A, G248P-1690I13, CR545463; Figure S13 in Additional data file 7).

\section{Quality control and analysis of new chromosome 22 sequences}

Although the long PCR systems we used utilize proofreading polymerases in combination with $T a q$ polymerase, they are still susceptible to base incorporation errors when compared to sequencing from bacterial clones. In addition, there is also the possibility that rearrangements could be introduced and propagated during the PCR process. To minimize the effect of base incorporation errors, we constructed libraries of each uncloned long PCR product directly in the sequencing vector and sequenced up to 20 -fold coverage. In addition, after generating finished sequence across the gaps, we analyzed that sequence in several ways to check for error. First we examined regions of overlap between sequence templates, which, therefore, had been sequenced twice from independent cloning events, either from long PCR product and bacterial clone (BAC, PAC or fosmid) or from two independent long PCR products. In the case of overlaps between long PCR derived sequences and bacterial clones, 11 base differences were identified out of 11,676 bp examined. Comparing overlapping long PCR products identified 27 base differences in 
(a)

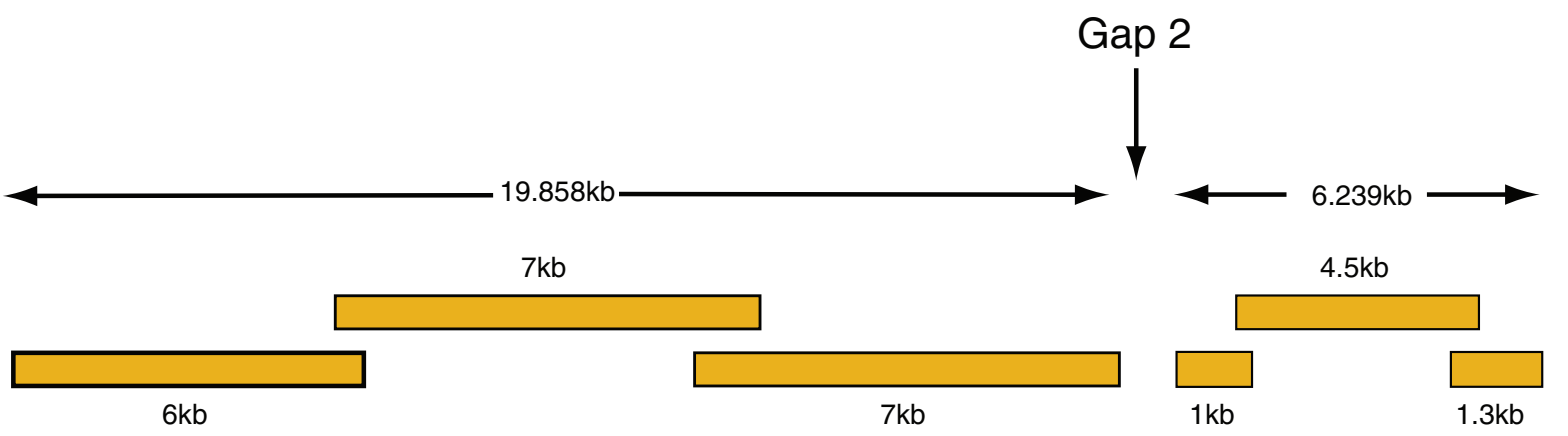

(b)

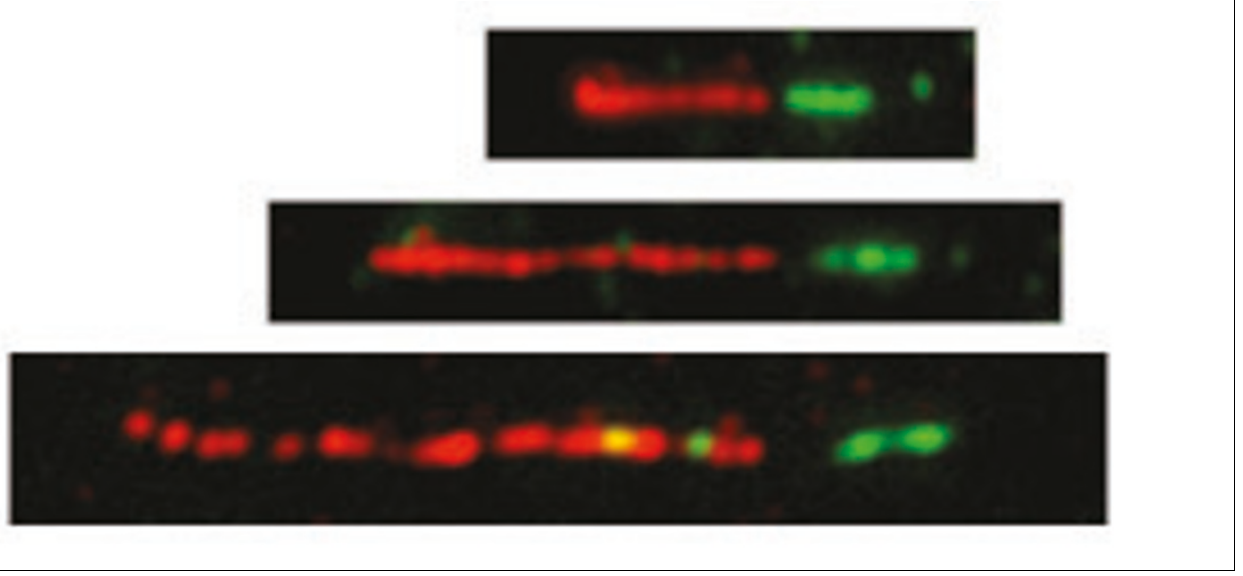

Figure 3

DNA fiber FISH using pools of long PCR products at gap 2 prior to final closure by long PCR. (a) Schematic representation of the three long PCR products from either side of the gap that were labeled and hybridized to DNA fibers. See Figure S5 in Additional data file 7 for identities. Note that nonrepetitive sequence represented $71 \%$ of $19,858 \mathrm{~kb}$ on the left-hand side of the gap and only $50 \%$ of $6,239 \mathrm{~kb}$ on the right-hand side, as determined by Repeatmasker. (b) Three example DNA fibers showing detection by FISH of hybridized centromeric and telomeric PCR product pools in red (Texas Red) and green (FITC), respectively. Estimation from the DNA fiber FISH images suggested that the remaining gap was $2-3 \mathrm{~kb}$ in size and this was closed by the PCR product c658c926rcL (sequence CU210860).

37,810 bp of sequence overlap. Both these base difference rates are consistent with the expected rates of variation due to sequence polymorphism between any two human genomes [27-29]. When we analyzed short PCR products representing 25 out of these 27 differences from 6 different individuals by single pass sequencing, only 2 were not either single nucleotide polymorphisms (19 out of 25) or short polymorphic runs of Ts (4 out of 25). Assuming these two base changes were sequencing errors and not rare polymorphisms, these data would give an error rate typical of finished quality sequence (2 out of $37,810 \mathrm{bp}$ ) [6,9]. We also sought additional confirmation of the quality of sequence produced from long PCR products by designing oligonucleotides from the long PCR sequence to generate an overlapping path of shorter PCR products $(<1,200 \mathrm{bp})$. Comparison of single pass sequencing of 219 PCR fragments with 84,546 bp of genomic sequence confirmed the integrity of the sequence in all cases. Base by base examination of $13,239 \mathrm{bp}$ of single pass sequence from one region (gap 3 ) identified only 2 possible errors, both of which were single base length difference in short repeat runs (poly $\mathrm{T}$ and poly CA), that could potentially be polymorphic, but which are also known 'difficult' sequences in single pass PCR sequencing. From these analyses we conclude that the sequence derived from the long PCR products is of a similar standard to conventional finished sequence from bacterial clones and the process was successful in closing recalcitrant gaps.

We subjected the new sequence from gaps 1-7 and the four deleted regions (approximately $820 \mathrm{~kb}$ ) to sequence analysis and gene annotation using our standard approach $[18,30]$ (additional annotation available at [26]). In total, 14 new coding genes, 2 non-coding genes (without an open reading frame of $>300 \mathrm{bp}$ ) and one pseudogene were annotated on to the chromosome 22 sequence including CYP2D6. No high identity matches ( $\geq 95 \%$ identity) were found to known human microRNA precursors. Seven of these annotations involved extension of genes that were partially annotated pre- 
viously [18] and extended into the previous gaps. One of the annotated genes is a partial structure. This gene annotation includes an additional 95 exons contributing 23,872 bp beyond the annotation described in [18], which included some annotations on added sequence. Therefore, addition of $820 \mathrm{~kb}(2.3 \%)$ of new chromosome 22 sequence within the previous gaps has increased the gene annotation by 1.6-1.8\% (by base content or exon count, respectively).

In order to try to understand whether particular phenomena underlie the persistent recalcitrance of certain genomic regions to sequencing, we re-examined the features of the sequences that surround and now close the gaps. At the telomeric boundary of the unclosed gap, gap 6, there is an $11.2 \mathrm{~kb}$ sequence consisting of $87 \%$ repetitive sequences and a GCrich (72\%) unique segment that has made it impossible with current sequencing technology to walk further into this gap. Analysis of the GC content of the new sequences revealed that five of the gaps have significantly higher GC content (mean GC ranging from $54.5-57.5 \%$ ) than the approximately $7.3 \mathrm{Mb}$ of $22 \mathrm{q} 13$ (mean GC $51 \%$ ) in which they reside ( $p<10^{-4}$, Wilcoxon rank sum test, $1 \mathrm{~kb}$ windows; Figure S14 in Additional data file 7). The two exceptions are gap 5, which was closed entirely with fosmids and has a GC content similar to the mean for $22 \mathrm{q} 13$ (51\%), and gap 1, with a GC content that is higher (mean 54\%) but differs less significantly from the surrounding sequence $(p<0.02)$. Given that chromosome 22 overall and 22q13 particularly have high GC content compared with the rest of the genome, it is clear that these sequences are unusual. Further analysis by sequence dot plot showed that several of the gaps also contain substantial simple tandem repeat sequences (Figure S15 in Additional data file 7). Comparing the density of tandem repeat bases per kilobase in $25 \mathrm{~kb}$ windows between the gap sequences and all chromosome 22 sequence indicated that the gaps are significantly enriched in tandem repeats ( $p=6.756 \mathrm{e}^{-10}$, Wilcoxon rank sum test, $25 \mathrm{~kb}$ windows). Taken together with the observation of the role of tandem repeats in the deletion of BAC CTA-437G10 (AL022330), it seems plausible that further investigation of the role of tandem repeats in these problem sequences is warranted.

\section{Conclusion}

Since the essential completion of the sequence of chromosome 22, 8 out of the 11 gaps have been closed by conventional mapping combined with novel PCR-based approaches. In total we generated $1.018 \mathrm{Mb}$ of new sequence to extend into and close these gaps, and the final sequences were incorporated into the latest version of the human chromosome 22 reference sequence; 95 additional exons were identified for 16 genes and one pseudogene, contributing an additional approximately $1.6 \%$ of gene annotation. In a parallel effort Bovee et al. [31] have used fosmid libraries generated from the DNA of multiple individuals to close 26 gaps across the genome, including identification of two fosmids across two of the gaps addressed here (gaps 2 and 7, referred to as 22_05 and 22_10 in Table 1 of [31]). These findings have confirmed the sequence additions reported here and, indeed utilized and built on the intermediate assemblies generated and released as part of this project. However, both studies illustrate the likely success of approaches that can be taken towards closing the remaining gaps in the human sequence. In the Bovee et al. study, a high throughput approach using new fosmid libraries was successful for $10 \%$ of the remaining gaps in the human genome. In this study we took a more exhaustive approach including fosmid libraries but also utilizing all available sources of sequence information in a PCR based strategy. In our case we were able to close $8(72.7 \%)$ out of the 11 remaining gaps on chromosome 22 studied, and all bar one of the gaps not associated with the LCR22 segmental duplications. Thus, it seems likely that the majority of gaps in the human genome will be tractable to closure strategies, some via initial high throughput approaches and the remainder by more detailed analysis. However, although high throughput approaches can be rapid, the more detailed analysis we have undertaken is not, as it involves multiple cycles of experimental work and decision-making, plus a degree of trial and error when amplifying by long PCR. Extending this approach genome-wide would require substantial investment in experienced genome mappers, unless the processes can be substantially streamlined. Alternatively, combining surface based or solution capture methods utilizing the sequences at the edge of gaps with high throughput sequencing (see, for instance, [32]) of the enriched sequence might provide a way into outstanding gaps. Hence, future progress on closure of gaps is likely to depend on the level of motivation that exists for the project or development of additional technologies.

In addition to closure of gaps, we also added $126 \mathrm{~kb}$ of sequence to patch 4 regions of the initial chromosome 22 sequence that had been identified as harboring deletions either as a result of polymorphisms, or clone artifacts. It is clear that providing these problem regions can be efficiently identified, it is possible to provide fixes. In these cases, one was identified from study of a known gene, two from analyzing the mapping of WIBR-2 fosmid end sequences and the other during routine mapping via DNA fiber FISH. Both fosmid end sequence mapping and DNA fiber FISH methods are unlikely to identify problems below $5-10 \mathrm{~kb}$ in size. Hence, small insertion/deletion problems (or polymorphisms) such as retrotransposon insertions will not be caught. In the future large-scale high throughput sequencing of many human genomes may provide resources that can be used to identify such insertion/deletion events. The question then will be whether it makes sense to maintain a single human reference sequence, and if so, to what criteria that reference should conform (for example, single haplotype, functional haplotypes, longest genome). The current reference sequence is a mosaic derived from more than eight individuals. However, approximately $70 \%$ of the reference assembly originated from a library of a single individual (the RPCI-11 BAC library). While 
it might be attractive to migrate the reference sequence towards defined haplotypes, this would be substantial additional work and in practice most regions are already likely to represent relatively common haplotypes because of the nature of human polymorphism [27,33,34].

Similarly, the availability of additional sequence of this type may provide sequence within current gaps. However, it is important to remember that in addition to the well mapped gaps residing in the 'euchromatin' of the human genome, there are many megabases of unknown human hetereochromatin still to be sequenced, including centromeres, the $\mathrm{p}$ arms of the acrocentric chromosomes, other heterochromatic blocks and some segmentally duplicated regions. We have provided some sequence for chromosome 22p, both as whole chromosome assembly [35] and as isolated BAC and fosmid clones (unpublished data list of chromosome 22p accessions in Additional data file 6). Placing new sequence into the increasingly small complement of euchromatic gaps will require substantial effort to verify its location, possibly using traditional mapping tools such as flow sorted chromosomes and somatic cell hybrids. In our opinion much mapping work remains to be done before we will see the complete human genome sequence.

\section{Materials and methods \\ Bacterial clone library screening and contig construction}

Conventional mapping of bacterial clones, PCR, hybridization and building of contigs was performed by standard protocols as described in $[4,36,37]$. Clone libraries used in this work are detailed in Additional data file 1.

\section{Sequencing}

Genomic sequencing of bacterial clones and sequencing of short PCR products was performed as previously described [38-40].

Long PCR products were sequenced using small insert libraries prepared from gel-purified PCR products [38]. Long PCR products were purified on low melting temperature agarose gels, sonicated and end repaired with mung bean nuclease. Small inserts of 300-500 bp and 500-800 bp were subcloned into pUC18 for sequencing and assembled using the PHRAP algorithm [38].

Sequence assemblies to form a golden path (agp) across new sequence and a whole chromosome sequence was performed as described in [41]. Additional data files 2 and 3 give details of positions of the gaps and deletions referred to here in multiple representative genome assemblies. Annotation of sequence was as described previously [30]. Tandem repeats were identified using tandem repeat finder [42]. Additional sequence processing and analysis was performed using custom perl scripts and R [43]. The agp file describing the chro- mosome 22 sequence assembly, the sequence in fasta format and the new and extended annotations in general feature format (GFF) are available at [26].

\section{Long PCR}

Long PCR primer pairs were designed using primer 3 [44] with specifications as recommended by the Roche Expand 20 $\mathrm{kb}+$ kit (catalogue number 1-811-002, Roche Diagnostics Ltd. Burgess Hill, UK). Most long-PCR amplifications were performed using Roche high molecular weight DNA (catalogue number 1-691-112), but in regions of repeated DNA sequences that proved difficult to assemble, additional PCR reactions from single individuals were amplified and sequenced. Long PCR was performed using either Roche Expand $20 \mathrm{~kb}+$ PCR system (initially Pwo/Taq enzyme combination prior to introduction of the Tgo/Taq version), Roche Expand Long Template-PCR System (catalogue number 1-681-842) or Novagen KOD Hot Start DNA polymerase kit (catalogue number 71086-4, Novagen (Merck Biosciences Ltd) Nottingham, UK). PCR conditions were as recommended by the manufacturers with the optional addition of between $1 \%$ and $6 \%$ dimethyl sulphoxide for some reactions. DNA (200-240 ng) was amplified in thin walled PCR tubes in $50 \mu \mathrm{l}$ reactions and at annealing temperatures of $62-65^{\circ} \mathrm{C}$ for 35 cycles. c658c926rcL (gap 2 final closure) included 1:1 7-deaza dGTP:dGTP, 3\% dimethyl sulphoxide and modified PCR cycle temperatures of $65^{\circ} \mathrm{C}$ annealing, $72^{\circ} \mathrm{C}$ extension (first two cycles) and $95^{\circ} \mathrm{C}$ denaturing.

\section{Standard PCR}

Standard PCR primers were designed using primer 3 [44] and amplified as described previously [39]. Primer design specifications were adjusted to take into account regions of high GC content and denaturing times/temperatures were increased. To generate the short PCR tiling paths and analyze possible polymorphisms, DNA samples from six individuals were amplified (three HapMap samples, NA07340, NA12873 and NA17119, plus NA06990, NA10847 and NA12873) in addition to the mixed Roche DNA employed to generate the long PCR products.

\section{Fiber FISH}

Fiber FISH and digital imaging essentially followed the procedure as described previously [45], with a slight modification in the preparation of DNA probes when PCR products were used. Briefly, in the case of gap 2 (Figure 3) three PCR products from each side of the gap were selected for FISH. PCR products were first cleaned using a GenElute ${ }^{\circledR}$ PCR Clean-Up kit (Sigma-Aldrich, Gillingham, Dorset, UK) and quantified on a NanoDrop ${ }^{\circledR}$ ND-100o spectrophotometer (Labtech International, Ringmer, East Sussex, UK). The cleaned PCR products from each side of the gap were pooled at equal molarity for each PCR product, and then labeled using either the Biotin-Nick Translation Mix and DIG-Nick Translation Mix (Roche Diagnostics, Burgess Hill, West Sussex, UK). Biotin-labeled probe was detected with Texas $\operatorname{Red}^{\circledR}$ 
conjugated avidin (Molecular Probes, Eugene, Oregon, USA). DIG-labeled probe with monocolonal mouse anti-dig antibody (Sigma-Aldrich) and FITC-conjugated goat anti-mouse antibody (Vector Laboratories, Orton Southgate, Peterborough, UK).

\section{Shotgun sequence assembly}

In the HapMap project, DNA samples of three individuals were flow sorted for chromosome 22 and 889,608 shotgun reads (approximately $2 \times$ read coverage for each individual) were generated mainly from plasmid libraries with an averaged insert size of 2.5 $\mathrm{Kb}$ [23]. The WCS dataset also included approximately 60,000 fosmid end sequences, separated by around $35^{-} 40 \mathrm{~kb}$, that were generated from a flow sorted chromosome 22 library [20]. To get a global sequence view of the whole of chromosome 22 and close possible gaps in 22q, a strategy was pursued to produce a shotgun assembly using all the HapMap reads including plasmids and fosmid sequences. The Phusion assembler was used to assemble the chromosome using WCS reads [46]. Aligning the contigs and scaffolds against the existing finished sequence, it was possible to identify the gaps where the reference sequence does not have coverage. The WCS assembly and HapMap reads are available for download at [47]. This was the main approach used for identifying sequence within gaps. In addition, to effectively separate novel contig sequences including gaps and 22p from the whole chromosome shotgun, we pursued a second strategy: using $22 \mathrm{q}$ finished sequences to guide the WCS assembly. Finished clone sequences were shredded into fragments with a read length of 1,000 bp and a paired insert size of $40,000 \mathrm{bp}$. The shredded reads accounted for about $2 \times$ coverage over 22q. Assigning the shredded reads back to 22q finished sequences, it was possible to build an assembly by removing those contigs that can be firmly placed on existing 22q sequence. It was expected that the assembly with mixed shredded data could be better than the pure WCS assembly in which the shotgun reads were from three individuals, and might place sequences extending immediately into the gaps at the borders of firmly mapped contigs. We have used this ' $22 \mathrm{p}$ assembly' (including any non-mapped sequence from 22p, 22 cen and 22q gaps) as a source of probes to screen for BAC clones in 22p (Additional data file 6). We have placed the mixed and 22p assemblies (including any non-mapped sequence) at [35] for any future applications.

\section{Abbreviations}

BAC, bacterial artificial chromosome; CNV, copy number variation; FISH, fluorescence in situ hybridization; HapMap, haplotype map; LCR, low copy repeat; PAC, P1 artificial chromosome; STS, sequence tagged site; WCS, whole chromosome shotgun sequence.

\section{Authors' contributions}

CGC carried out long PCR gap filling and sequence validation experiments and analysis. OTM carried out bacterial clone library screening, map building by restriction enzyme fingerprinting, long PCR and fiber FISH. JEC and DMB carried out gene annotation of the novel sequences. KO, DW, KM, and JR were responsible for library preparation and DNA sequencing of gap clones and PCR products. SMG and FY supervised and conducted DNA fiber FISH experiments. DMB carried out sequence analysis, sequence assemblies and management of AGP and tpf infrastructure. ID designed and supervised the project, conducted sequence analysis of gap sequences and assembled figures and tables. ID and CGC prepared the manuscript. All authors have read and approved the final manuscript.

\section{Additional data files}

The following additional data are available with the online version of this paper. Additional data file 1 is a table listing the clone libraries used in this work. Additional data file 2 is a table listing the coordinates of the gaps and deletions addressed in this work on numerous genome builds. In addition, this file contains the tile path file (tpf) specification for chromosome 22. Additional data file 3 contains the Additional data file 1 table and the tpf file in tab delimited text format. Additional data file 4 is a table listing the STSs used in clone library screening as referred to in Figures S4-S13. Additional data file 5 is a table listing the long PCR products generated. Additional data file 6 is a table listing clones identified as mapping to human chromosome 22p or 22cen. Additional data file 7 contains Figures S1-S15.

\section{Acknowledgements}

Thank you to Bruce Roe and his group for sharing mapping information on two gaps in 22ql (gaps $C$ and D). This work was supported by the Wellcome Trust.

\section{References}

I. International Human Genome Sequencing Consortium: Finishing the euchromatic sequence of the human genome. Nature 2004, 43 I:93I-945

2. Venter JC, Adams MD, Myers EW, Li PW, Mural RJ, Sutton GG, Smith $\mathrm{HO}$, Yandell M, Evans CA, Holt RA, Gocayne JD, Amanatides P, Ballew RM, Huson DH, Wortman JR, Zhang Q, Kodira CD, Zheng XH, Chen L, Skupski M, Subramanian G, Thomas PD, Zhang J, Gabor Miklos GL, Nelson C, Broder S, Clark AG, Nadeau J, McKusick VA, Zinder $N$, et al:: The sequence of the human genome. Science 200|, 29 I: |304-|35|.

3. International Human Genome Sequencing Consortium: Initial sequencing and analysis of the human genome. Nature 200I, 409:860-921.

4. Bentley DR, Deloukas P, Dunham A, French L, Gregory SG, Humphray S], Mungall AJ, Ross MT, Carter NP, Dunham I, Scott CE, Ashcroft KJ, Atkinson AL, Aubin K, Beare DM, Bethel G, Brady N, Brook JC, Burford DC, Burrill WD, Burrows C, Butler AP, Carder C, Catanese J], Clee CM, Clegg SM, Cobley V, Coffey AJ, Cole CG, Collins JE, et al:: The physical maps for sequencing human chromosomes I, 6, 9, 10, 1 3, 20 and X. Nature 2001, 409:942-943.

5. McPherson JD, Marra M, Hillier L, Waterston RH, Chinwalla A, Wallis J, Sekhon M, Wylie K, Mardis ER, Wilson RK, Fulton R, Kucaba TA, 
Wagner-McPherson C, Barbazuk WB, Gregory SG, Humphray SJ, French L, Evans RS, Bethel G, Whittaker A, Holden JL, McCann OT, Dunham A, Soderlund C, Scott CE, Bentley DR, Schuler G, Chen HC Jang W, Green ED, et al.: A physical map of the human genome. Nature 200I, 409:934-94I.

6. Schmutz J, Wheeler J, Grimwood J, Dickson M, Yang J, Caoile C, Bajorek E, Black S, Chan YM, Denys M, Escobar J, Flowers D, Fotopulos D, Garcia C, Gomez M, Gonzales E, Haydu L, Lopez F, Ramirez L, Retterer J, Rodriguez A, Rogers S, Salazar A, Tsai M, Myers RM: Quality assessment of the human genome sequence. Nature 2004, 429:365-368.

7. Bailey JA, Gu Z, Clark RA, Reinert K, Samonte RV, Schwartz S, Adams MD, Myers EW, Li PW, Eichler EE: Recent segmental duplications in the human genome. Science 2002, 297:1003-1007.

8. Dunham I, Hunt AR, Collins JE, Bruskiewich R, Beare DM, Clamp M, Smink LJ, Ainscough R, Almeida JP, Babbage A, Bagguley C, Bailey J, Barlow K, Bates KN, Beasley O, Bird CP, Blakey S, Bridgeman AM, Buck D, Burgess J, Burrill WD, O'Brien KP, et al.: The DNA sequence of human chromosome 22. Nature 1999, 402:489-495.

9. Felsenfeld A, Peterson J, Schloss J, Guyer M: Assessing the quality of the DNA sequence from the Human Genome Project. Genome Res 1999, 9:1-4.

10. Idle JR, Corchero J, Gonzalez FJ: Medical implications of HGP's sequence of chromosome 22. Lancet 2000, 355:319.

II. Stewart CA, Horton R, Allcock RJ, Ashurst JL, Atrazhev AM, Coggill P, Dunham I, Forbes S, Halls K, Howson JM, Humphray SJ, Hunt S, Mungall AJ, Osoegawa K, Palmer S, Roberts AN, Rogers J, Sims S, Wang Y, Wilming LG, Elliott JF, de Jong PJ, Sawcer S, Todd JA, Trowsdale J, Beck S: Complete MHC haplotype sequencing for common disease gene mapping. Genome Res 2004, I 4: I I76-I I87.

12. Edelmann L, Pandita RK, Spiteri E, Funke B, Goldberg R, Palanisamy N, Chaganti RS, Magenis E, Shprintzen RJ, Morrow BE: A common molecular basis for rearrangement disorders on chromosome 22q I I. Hum Mol Genet 1999, 8: I I57-II67.

13. Collins JE, Mungall AJ, Badcock KL, Fay JM, Dunham I: The organization of the gamma-glutamyl transferase genes and other low copy repeats in human chromosome 22q I I. Genome Res | 1997, 7:522-531.

14. Shaikh TH, Kurahashi H, Saitta SC, O'Hare AM, Hu P, Roe BA, Driscoll DA, McDonald-McGinn DM, Zackai EH, Budarf ML, Emanuel BS: Chromosome 22-specific low copy repeats and the 22q II.2 deletion syndrome: genomic organization and deletion endpoint analysis. Hum Mol Genet 2000, 9:489-50I.

15. Redon R, Ishikawa S, Fitch KR, Feuk L, Perry GH, Andrews TD, Fiegler H, Shapero MH, Carson AR, Chen W, Cho EK, Dallaire S, Freeman JL, Gonzalez JR, Gratacos M, Huang J, Kalaitzopoulos D, Komura D, MacDonald JR, Marshall CR, Mei R, Montgomery L, Nishimura K, Okamura K, Shen F, Somerville MJ, Tchinda J, Valsesia A, Woodwark $\mathrm{C}$, Yang F, et al:: Global variation in copy number in the human genome. Nature 2006, 444:444-454.

16. Tuzun E, Sharp AJ, Bailey JA, Kaul R, Morrison VA, Pertz LM, Haugen E, Hayden H, Albertson D, Pinkel D, Olson MV, Eichler EE: Finescale structural variation of the human genome. Nat Genet 2005, 37:727-732.

17. Copy Number Variation Project [http://www.sanger.ac.uk/ humgen/cnv/data/cnv_data/]

18. Collins JE, Goward ME, Cole CG, Smink LJ, Huckle EJ, Knowles S, Bye JM, Beare DM, Dunham I: Reevaluating human gene annotation: a second-generation analysis of chromosome 22. Genome Res $2003,13: 27-36$

19. Kim UJ, Shizuya H, de Jong PJ, Birren B, Simon MI: Stable propagation of cosmid sized human DNA inserts in an $F$ factor based vector. Nucleic Acids Res 1992, 20: 1083-1085.

20. Kim UJ, Shizuya H, Sainz J, Garnes J, Pulst SM, de Jong P, Simon MI: Construction and utility of a human chromosome 22-specific Fosmid library. Genet Anal 1995, I 2:8I-84.

21. NCBI Trace Server [http://www.ncbi.nih.gov/Traces/trace.cgi]

22. Chimpanzee Sequencing and Analysis Consortium.: Initial sequence of the chimpanzee genome and comparison with the human genome. Nature 2005, 437:69-87.

23. International HapMap Consortium: The International HapMap Project. Nature 2003, 426:789-796.

24. Chr 22 WCS ftp data [ftp://ftp.sanger.ac.uk/pub/zn I/chr22/]

25. Istrail S, Sutton GG, Florea L, Halpern AL, Mobarry CM, Lippert R, Walenz B, Shatkay H, Dew I, Miller JR, Flanigan MJ, Edwards NJ, Bolanos R, Fasulo D, Halldorsson BV, Hannenhalli S, Turner R, Yooseph S, Lu F, Nusskern DR, Shue BC, Zheng XH, Zhong F,
Delcher AL, Huson DH, Kravitz SA, Mouchard L, Reinert K, Remington KA, Clark AG, et al.: Whole-genome shotgun assembly and comparison of human genome assemblies. Proc Natl Acad Sci USA 2004, 101:1916-1921.

26. Human Chromosome 22 Gap Closure Project Overview [http://www.sanger.ac.uk/HGP/Chr22/GapClosure/]

27. Dawson E, Chen Y, Hunt S, Smink LJ, Hunt A, Rice K, Livingston S, Bumpstead S, Bruskiewich R, Sham P, Ganske R, Adams M, Kawasaki K, Shimizu N, Minoshima S, Roe B, Bentley D, Dunham I: A SNP resource for human chromosome 22: extracting dense clusters of SNPs from the genomic sequence. Genome Res 200I, II:170-178.

28. Sachidanandam R, Weissman D, Schmidt SC, Kakol JM, Stein LD, Marth G, Sherry S, Mullikin JC, Mortimore B], Willey DL, Hunt SE, Cole CG, Coggill PC, Rice CM, Ning Z, Rogers J, Bentley DR, Kwok PY, Mardis ER, Yeh RT, Schultz B, Cook L, Davenport R, Dante M, Fulton L, Hillier L, Waterston RH, McPherson JD, Gilman B, Schaffner $S$, et al.: A map of human genome sequence variation containing 1.42 million single nucleotide polymorphisms. Nature 200I, 409:928-933.

29. Taillon-Miller P, Gu Z, Li Q, Hillier L, Kwok PY: Overlapping genomic sequences: a treasure trove of single-nucleotide polymorphisms. Genome Res 1998, 8:748-754.

30. Ashurst JL, Collins JE: Gene annotation: prediction and testing. Annu Rev Genomics Hum Genet 2003, 4:69-88.

31. Bovee D, Zhou Y, Haugen E, Wu Z, Hayden HS, Gillett W, Tuzun E, Cooper GM, Sampas N, Phelps K, Levy R, Morrison VA, Sprague J, Jewett D, Buckley D, Subramaniam S, Chang J, Smith DR, Olson MV, Eichler EE, Kaul R: Closing gaps in the human genome with fosmid resources generated from multiple individuals. Nat Genet 2008, 40:96-101.

32. Albert TJ, Molla MN, Muzny DM, Nazareth L, Wheeler D, Song $X$, Richmond TA, Middle CM, Rodesch MJ, Packard CJ, Weinstock GM, Gibbs RA: Direct selection of human genomic loci by microarray hybridization. Nat Methods 2007, 4:903-905.

33. Gabriel SB, Schaffner SF, Nguyen H, Moore JM, Roy J, Blumenstiel B, Higgins J, DeFelice M, Lochner A, Faggart M, Liu-Cordero SN, Rotimi C, Adeyemo A, Cooper R, Ward R, Lander ES, Daly MJ, Altshuler D: The structure of haplotype blocks in the human genome. Science 2002, 296:2225-2229.

34. International HapMap Consortium: A haplotype map of the human genome. Nature 2005, 437:1299-1320.

35. Chr 22 WCS ftp data: mixed assembly [ftp://ftp.sanger.ac.uk/ pub/znI/chr22/assembly_mix]

36. Dunham I, Dewar K, Kim U-J, Ross MT: Bacterial cloning systems. In Genome Analysis: A Laboratory Manual Series, Cloning Systems Volume 3. Edited by: Birren B, Green ED, Klapholz S, Myers RM, Riethman H, Roskams J. Cold Spring Harbor, NY: Cold Spring Harbor Laboratory Press; 1999: I-86.

37. Mungall AJ, Humphray S): Assembling physical maps and sequence clone selection. In Genome Mapping and Sequencing Edited by: Dunham I. Wymondham: Horizon Scientific Press; 2003:167-200.

38. Hunt $A R$, Willey $D L$, Quail MA: Finishing genomic sequence and dealing with problem sequences. In Genome Mapping and Sequencing Edited by: Dunham I. Wymondham: Horizon Scientific Press; 2003:3।5-355.

39. Collins JE, Wright CL, Edwards CA, Davis MP, Grinham JA, Cole CG, Goward ME, Aguado B, Mallya M, Mokrab Y, Huckle EJ, Beare DM, Dunham I: A genome annotation-driven approach to cloning the human ORFeome. Genome Biol 2004, 5:R84.

40. Jones MC, Sims SK: Shotgun sequencing. In Genome Mapping and Sequencing Edited by: Dunham I. Wymondham: Horizon Scientific Press; 2003:279-313.

4I. Collins JE, Beare DM: Annotating mammalian genome sequence. In Genome Mapping and Sequencing Edited by: Dunham I. Wymondham: Horizon Scientific Press; 2003:397-434.

42. Benson G: Tandem repeats finder: a program to analyze DNA sequences. Nucleic Acids Res 1999, 27:573-580.

43. Ihaka R, Gentleman R: R: a language for data analysis and graphics. J Computational Graphical Stat 1996, 5:299-314.

44. Rozen S, Skaletsky H: Primer3 on the WWW for general users and for biologist programmers. Methods Mol Biol 2000, 132:365-386.

45. Korbel JO, Urban AE, Affourtit JP, Godwin B, Grubert F, Simons JF, Kim PM, Palejev D, Carriero NJ, Du L, Taillon BE, Chen Z, Tanzer A, Saunders AC, Chi J, Yang F, Carter NP, Hurles ME, Weissman SM, Harkins TT, Gerstein MB, Egholm M, Snyder M: Paired-end 
mapping reveals extensive structural variation in the human genome. Science 2007, 3 18:420-426.

46. Mullikin JC, Ning Z: The phusion assembler. Genome Res 2003, | 3:8|-90.

47. Chr 22 WCS ftp data: wcs assembly [ftp://ftp.sanger.ac.uk/pub/ $\mathrm{znI}$ //chr22/assembly_wcs]

48. Sonnhammer EL, Durbin R: A dot-matrix program with dynamic threshold control suited for genomic DNA and protein sequence analysis. Gene 1995, 167:GCI-10.

49. chr22.agp [http://www.sanger.ac.uk/HGP/Chr22/GapClosure/ chr22.agp] 